\title{
WHO-defined 'myelodysplastic syndrome with isolated del(5q)' in 88 consecutive patients: survival data, leukemic transformation rates and prevalence of JAK2, MPL and IDH mutations
}

\author{
MM Patnaik ${ }^{1}, \mathrm{TL}_{\text {Lasho }}{ }^{1}, \mathrm{CM}$ Finke ${ }^{1}, \mathrm{~N}$ Gangat $^{1}$, D Caramazza ${ }^{2}$, SG Holtan ${ }^{1}$, A Pardanani $^{1}$, RA Knudson ${ }^{3}$, RP Ketterling $^{3}$, \\ D Chen ${ }^{4}$, JD Hoyer ${ }^{4}$, CA Hanson ${ }^{4}$ and A Tefferi ${ }^{1}$ \\ ${ }^{1}$ Division of Hematology, Department of Medicine, Mayo Clinic, Rochester, MN, USA; ${ }^{2}$ Cattedra ed U.O. di Ematologia, \\ Policlinico Universitario di Palermo, Palermo, Italy; ${ }^{3}$ Division of Cytogenetics, Department of Laboratory Medicine, Mayo Clinic, \\ Rochester, MN, USA and ${ }^{4}$ Division of Hematopathology, Department of Laboratory Medicine, Mayo Clinic, Rochester, MN, USA
}

\begin{abstract}
The 2008 World Health Organization (WHO) criteria were used to identify 88 consecutive Mayo Clinic patients with 'myelodysplastic syndrome with isolated del(5q)' (median age $\mathbf{7 4}$ years; 60 females). In all, $60(68 \%)$ patients were followed up to the time of their death. Overall median survival was 66 months; leukemic transformation was documented in five $(5.7 \%)$ cases. Multivariable analysis identified age $\geqslant 70$ years $(P=0.01)$, transfusion need at diagnosis $(P=0.04)$ and dysgranulopoiesis $(P=0.02)$ as independent predictors of shortened survival; the presence of zero (low risk), one (intermediate risk) or $\geqslant 2$ (high risk) risk factors corresponded to median survivals of 102,52 and 27 months, respectively. Janus kinase 2 (JAK2), thrombopoietin receptor (MPL), isocitrate dehydrogenase 1 (IDH1) and IDH2 mutational analysis was performed on archived bone marrows in 78 patients; JAK2V617F and MPLW515L mutations were shown in five $(6.4 \%)$ and three $(3.8 \%)$ patients, respectively, and did not seem to affect phenotype or prognosis. IDH mutations were not detected. Survival was not affected by serum ferritin and there were no instances of death directly related to iron overload. The current study is unique in its strict adherence to WHO criteria for selecting study patients and providing information on long-term survival, practical prognostic factors, baseline risk of leukemic transformation and the prevalence of JAK2, MPL and IDH mutations.
\end{abstract}

Leukemia (2010) 24, 1283-1289; doi:10.1038/leu.2010.105;

published online 20 May 2010

Keywords: prognosis; 5q-; isocitrate dehydrogenase; ferritin; iron

\section{Introduction}

'Myelodysplastic syndrome with isolated del $(5 q)$ ' constitutes one of seven World Health Organization (WHO)-defined categories of adult myelodysplastic syndromes (MDS). ${ }^{1}$ It is to be recalled that the traditional definition of 'the $5 q-$ syndrome' stipulated a phenotype characterized by macrocytic anemia, erythroid hypoplasia, normal or elevated platelet count, hypolobulated megakaryocytes and isolated del $(5 q) .{ }^{2}$ However, not all cases of del $(5 q)$-associated MDS variants fit neatly into this original description and can be histologically and cytogenetically diverse. For example, in a recent report of $130 \mathrm{MDS}$ patients who had del $(5 q)$ as part of their karyotype, only $6(5 \%)$ met the classical description of the ' $5 q-$ syndrome'. $^{3}$ The

Correspondence: Professor A Tefferi, Division of Hematology, Department of Medicine, Mayo Clinic, 200 First Street, SW, Rochester, MN 55905, USA. E-mail: tefferi.ayalew@mayo.edu

We dedicate the current study to our beloved friend and colleague, Gordon W Dewald, PhD, (1943-2010) who has left a legacy of excellence and superior leadership in clinical cytogenetics at the Mayo Clinic.

Received 12 March 2010; accepted 30 March 2010; published online 20 May 2010 current WHO definition for 'MDS with isolated del $(5 q)$ ' has clarified the diagnostic criteria and does not specify megakaryocyte morphology, extent of trilineage dysplasia or ring sideroblast count, but requires the presence of an isolated $\operatorname{del}(5 q)$, a blast count of $<5 \%$ in the bone marrow (BM) and $<1 \%$ in the peripheral blood (PB) and absence of Auer rods. ${ }^{1}$

Very few published studies on ' $5 q-$ syndrome' or del(5q)associated MDS follow standard criteria for patient selection; it is, therefore, not surprising that the reported median survival figures range from 28 months to 12 years and leukemic transformation rates from 7 to $16 \% .^{3-8}$ Isolated del(5q) abnormality can occur in both acute and chronic myeloid malignancies including myeloproliferative neoplasms (MPNs). ${ }^{9,10}$ Furthermore, among MDS patients with del $(5 q)$, most do not meet the WHO criteria for classification under the category of 'MDS with isolated del $(5 q)$ '. For example, in the aforementioned study of 130 patients with del( $5 q)$-associated MDS, 32 (25\%) patients had therapy-related MDS, $52(40 \%)$ had refractory anemia with excess blast, $19(15 \%)$ had unclassifiable myelodysplastic syndrome and the majority had additional cytogenetic abnormalities. $^{3}$ Similarly, the number of patients that are potentially classifiable as 'MDS with isolated $\operatorname{del}(5 q)^{\prime}$ in other studies constitutes only a fraction of their study population and the sample size for informative patients rarely exceeds 50 patients. Therefore, it has been difficult to discern the true natural history of patients with 'MDS with isolated del(5q)' and an accurate account of their molecular phenotype. It is with this background that we report on mature survival data and information on Janus kinase 2 (JAK2), thrombopoietin receptor (MPL) and isocitrate dehydrogenase $(I D H)$ mutational frequencies among 88 consecutive patients who clearly met the 2008 WHO diagnostic criteria for 'MDS with isolated $\operatorname{del}(5 q)$ '.

\section{Materials and methods}

After obtaining approval by the Mayo Clinic institutional review board, we queried an institutional cytogenetic database from 1989 to 2009, in order to identify patients whose BM karyotype analysis revealed at least two metaphases with isolated deletions of chromosome 5q. Medical records, BM and cytogenetic specimens were reviewed in all cases to ensure accuracy of diagnosis. Only those patients who met the $2008 \mathrm{WHO}$ criteria for 'MDS with isolated del $(5 q)$ ' were included in the data analysis. ${ }^{1}$ In patients who were alive, every attempt was made to update follow-up information, by means of a questionnaire/ telephone call sent to both patients and their primary doctors, 
and the 'date of last contact' reflected this time point and not the last time they were seen at the Mayo Clinic.

\section{Morphology}

Morphological evaluation of the BM and PB was performed according to the standard evaluation protocol at the Mayo Clinic. All 88 cases in the current study had their BM examination and cytogenetic studies performed and the diagnosis was reported at our institution. In addition, BM and PB slides were available for re-review in 80 patients. Dysplasia was recorded only if the dysplastic features within one cell line constituted more than $10 \%$ of the cell line examined. Dyserythropoiesis was defined by the presence of nuclearcytoplasmic asynchrony, multinuclearity, nuclear fragmentation, internuclear bridging or irregular nuclear outlines. Dysgranulopoiesis was defined by the presence of nuclear hypolobation (for example, pseudo-Pleger-Huet anomaly), cytoplasmic hypogranulation, atypical nuclear segmentation or significant dual butyrate and chloroacetate esterase-positive myeloid precursor cells. Megakaryocytic dysplasia was defined by the presence of micromegakaryocytes and megakaryocytes with nonlobated and hypolobated nuclei.

\section{Cytogenetics and molecular studies}

Cytogenetic studies were performed using short-term BM cultures along with conventional G- or Q-banding fluorescence. $^{11}$ When possible, a total of 20 metaphases were evaluated. The findings were described according to the International Society of Cytogenetic Nomenclature. ${ }^{12}$ Archived BM cell pellets were stored at $-70{ }^{\circ} \mathrm{C}$ in methanol:glacial acetic acid (2:1) fixative. After appropriate washing techniques, the DNA on these patients was extracted by using the Qiagen DNA Mini extraction kit (Qiagen, Santa Clarita, CA, USA) and analyzed for JAK2V617F and MPL (exon 10) mutations, by using previously described methods. ${ }^{13,14}$

$I D H 1$ and $I D H 2$ mutation analysis was performed by direct nucleotide sequencing. The primer sequences used to amplify IDH1 exon 4 were: 5'-CGGTCTTCAGAGAAGCCATT-3' (sense) and 5'-CACATTATTGCCAACATGAC-3' (anti-sense), as previously described..$^{15} \mathrm{IDH} 2$ was amplified using $5^{\prime}$-CCACTATTA TCTCTGTCCTC-3' (sense) and 5'-GCTAGGCGAGGAGCTCCA GT-3' (anti-sense) primers, as previously described. ${ }^{16}$ Briefly, both reactions were performed in $25 \mu$ volume, containing $100 \mathrm{ng}$ of DNA, $0.25 \cup$ Taq polymerase, $0.3 \mathrm{~mm}$ each of dATP, dCTP, dGTP and dTTP, $5 \mu$ l of a $10 \times$ PCR buffer (Roche Diagnostics, Indianapolis, IN, USA) and $0.2 \mu \mathrm{M}$ each of sense and anti-sense primers. The reaction was denatured at $94^{\circ} \mathrm{C}$ for $3 \mathrm{~min}$ followed by 35 cycles of denaturing at $94^{\circ} \mathrm{C}$ for $30 \mathrm{~s}$, annealing at $57^{\circ} \mathrm{C}$ for $30 \mathrm{~s}$ and extension at $72{ }^{\circ} \mathrm{C}$ for $40 \mathrm{~s}$. After a final extension at $72{ }^{\circ} \mathrm{C}$ for $2 \mathrm{~min}$, the products were confirmed by running on $1.3 \%$ agarose gel and purified using Qiagen PCR Quick Purification Kit (Qiagen). The product was submitted to automated sequencing by ABI PRISM 3730xl analyzer (Applied Biosystems Inc, Foster City, CA, USA) to screen for the presence of mutations.

\section{Statistical analysis}

Descriptive and statistically analyzed data were based on parameters collected at the time of initial diagnosis. Statistical procedures used were conventional and all data were analyzed by using StatView (SAS Institute, Cary, NC, USA). All P-values were two-tailed and statistical significance was set at the level of $P<0.05$. Continuous variables were summarized as medians and ranges. Categorical variables were described as count and relative frequency. Comparison between categorical variables was performed by $\chi^{2}$-statistics. Comparison of continuous variables between categories was performed by either MannWhitney U-test or Kruskal-Wallis test. Survival analysis was performed by the Kaplan-Meier method taking the interval from the date of diagnosis to death or last contact. The log-rank test was used to compare survival data. Cox regression model was used for multivariable analysis.

\section{Results}

\section{Clinical, histological and cytogenetic details at presentation}

Over 24000 unique patient cytogenetic studies performed at our institution, mainly between 1989 and 2009, were screened and $190(\sim 1 \%)$ patients with isolated del(5q) were identified. Table 1 lists the spectrum of hematological disorders associated with an isolated $\operatorname{del}(5 q)$ abnormality, and also provides information on their JAK2V617F mutational status and subsequent disease transformation into acute myeloid leukemia (AML) or a more aggressive disease phenotype. Among these 190 patients with isolated $\operatorname{del}(5 q), 88(\sim 46 \%)$ met the 2008 WHO criteria for 'MDS with isolated del $(5 q)$ '. The median age for this cohort was 74 years (range, 28-89; 60 females) and $61(69 \%)$ patients were transfusion dependent at the time of diagnosis. The main clinical and laboratory features of these 88 patients, stratified by the presence or absence of the JAK2V617F mutation, are outlined in Table 2. Cytogenetic data were available for re-review in all 88 cases, and BM and PB specimens were available for re-review in 80 patients; Table 3 describes their pathological and cytogenetic details.

\section{Molecular studies}

Archived BM was available for JAK2, MPL and IDH mutation analysis in 78 patients; $5(6.4 \%)$ patients harbored the JAK2 V617F mutation (JAK2V617F allele burden ranged from 1 to $10 \%)$ and $3(\sim 4 \%)$ patients harbored the MPLW515L mutation. One patient carried both mutations. Neither IDH1 nor $I D H 2$ mutations were detected in any patient. As illustrated in Table 2, there were no significant differences between JAK2V617F-positive and -negative cases in terms of age, hemoglobin level, leukocyte count, platelet count or clinical outcome. Ring sideroblasts were not seen in four of the five JAK2-mutated cases. Table 4 provides clinical and BM morphology details of the three patients with MPL mutations; none of these three patients displayed thrombocytosis and their survival did not seem to be compromised by the presence of the mutation (Table 4).

\section{Comorbid conditions and treatment}

Eight patients had coexisting hematological disorders including two with chronic lymphocytic leukemia, two monoclonal gammopathy of undetermined significance, two multiple myeloma and one paroxysmal nocturnal hemoglobinuria. In total, 47 (53\%) patients were prescribed drug therapy in an attempt to ameliorate anemia: erythropoiesis-stimulating agents in $28(32 \%)$ patients, lenalidomide in $18(20 \%)$ and thalidomide, azacytidine and danazol in one patient each. Of the 18 patients who received lenalidomide therapy, 5 (28\%) achieved transfusion independence, $5(28 \%)$ had a decrease in their transfusion requirement and 8 (44\%) showed no response. None of the five 
Table 1 Spectrum of hematological disorders associated with isolated del(5q) and information on their JAK2V617F mutational status and subsequent disease transformation

\begin{tabular}{|c|c|c|c|c|c|}
\hline Diagnosis & $\begin{array}{l}\text { JAK2V617F negative } \\
\qquad(\mathrm{n}=142)\end{array}$ & $\begin{array}{c}\text { JAK2V617F } \\
\text { positive }(\mathrm{n}=16)\end{array}$ & $\begin{array}{c}\text { JAK2V617F } \\
\text { unknown }(\mathrm{n}=32)\end{array}$ & $\begin{array}{c}\text { Total }(\mathrm{n}=190) \\
(\%)\end{array}$ & $\begin{array}{l}\text { Subsequent disease } \\
\text { transformation (n) }\end{array}$ \\
\hline $\begin{array}{l}\text { WHO-defined 'MDS } \\
\text { with isolated del( }(5 q) \text { ' }\end{array}$ & 78 & 5 & 5 & $88(46)$ & $\begin{array}{l}\text { AML (5) } \\
\text { RAEB-1 (1) } \\
\text { CMML (1) }\end{array}$ \\
\hline RAEB-1 & 10 & 1 & 2 & $13(7)$ & AML (1) \\
\hline RAEB-2 & 11 & 1 & 3 & $15(8)$ & AML (4) \\
\hline MDS-U & 0 & 0 & 2 & $2(1)$ & \\
\hline CMML & 2 & 0 & 2 & $4(2)$ & \\
\hline RARS-T & 0 & 1 & 0 & $1(0.5)$ & \\
\hline MDS/MPN overlap & 1 & 0 & 1 & $2(1)$ & \\
\hline PV & 0 & 5 & 2 & 7 (4) & Post-PV MF (3) \\
\hline ET & 0 & 1 & 1 & $2(1)$ & \\
\hline PMF & 2 & 1 & 2 & $5(3)$ & AML (1) \\
\hline MPN-U & 2 & 1 & 1 & $4(2)$ & \\
\hline Systemic mastocytosis & 1 & 0 & 0 & $1(0.5)$ & \\
\hline AML & 16 & 0 & 6 & $22(11)$ & NA \\
\hline Others $^{a}$ & 18 & 0 & 5 & $23(12)$ & \\
\hline
\end{tabular}

Abbreviations: AML, acute myeloid leukemia; CMML, chronic myelomonocytic leukemia; ET, essential thrombocytosis; JAK2, janus kinase 2; MDS, myelodysplastic syndrome; MDS-U, MDS unclassifiable; MPN, myeloproliferative neoplasm; MPN-U, MPN unclassifiable; NA, not applicable; PMF, primary myelofibrosis; PV, polycythemia vera; RAEB, refractory anemia with excess blasts; RARS-T, refractory anemia with ringed sideroblasts with thrombocytosis; WHO, world health organization.

aOthers include patients with plasma cell dyscrasias and lymphoproliferative disorders that were noted to have an isolated del 5(q) on cytogenetics, with no overt features of myelodysplasia based on bone marrow morphology.

Table 2 Clinical and laboratory features of 88 patients with the World Health Organization (WHO)-defined 'myelodysplastic syndrome with isolated del $(5 q)^{\prime}$, stratified by the presence or absence of the JAK2V617F mutation

\begin{tabular}{|c|c|c|c|c|c|}
\hline Variable & $\begin{array}{l}\text { Entire cohort } \\
\quad(\mathrm{n}=88)\end{array}$ & $\begin{array}{l}\text { JAK2V617F } \\
\text { negative }(\mathrm{n}=78)\end{array}$ & $\begin{array}{l}\text { JAK2V617F } \\
\text { positive }(\mathrm{n}=5)\end{array}$ & $\begin{array}{l}\text { JAK2V617F } \\
\text { unknown }(\mathrm{n}=5)\end{array}$ & $\mathrm{P}^{\mathrm{a}}$-value \\
\hline Age in years (median and range) & $74(28-89)$ & $74(28-89)$ & $74(70-80)$ & $79(70-81)$ & 0.54 \\
\hline Sex, M/F & $28 / 60$ & $23 / 55$ & $3 / 2$ & $2 / 3$ & 0.15 \\
\hline $\begin{array}{l}\text { Hemoglobin in g per } 100 \mathrm{ml} \\
\text { (median and range) }\end{array}$ & $9.2(4.7-13.3)$ & $9.3(4.7-13.3)$ & $9.1(5.4-10.6)$ & $9.0(8.2-9.2)$ & 0.32 \\
\hline MCV in fl (median and range) & $103(79-130)$ & $102(79-130)$ & $102(92-116)$ & $104(98-111)$ & 0.45 \\
\hline $\begin{array}{l}\text { Leukocyte count } \times 10^{9} \text { cells per I } \\
\text { (median and range) }\end{array}$ & $4.7(1.5-27.3)$ & $4.7(1.5-27.3)$ & $4.5(3.5-10.7)$ & $5.1(3-7.8)$ & 0.98 \\
\hline $\begin{array}{l}\text { Absolute neutrophil count } \times 10^{9} \text { cells } \\
\text { per I (median and range) }\end{array}$ & $2.4(0.5-10.5)$ & $2.5(0.5-10.5)$ & $2.1(1.7-7.0)$ & $3.2(1.9-4.1)$ & 0.54 \\
\hline $\begin{array}{l}\text { Absolute lymphocyte count } \times 10^{9} \text { cells } \\
\text { per I (median and range) }\end{array}$ & $1.4(0.25-18.5)$ & $1.3(0.25-18.5)$ & $1.6(1.2-2.1)$ & $1.4(0.6-2.3)$ & 0.67 \\
\hline $\begin{array}{l}\text { Platelet count } \times 10^{9} \text { cells per I } \\
\text { (median and range) }\end{array}$ & $235(47-1800)$ & 230(52-780) & $221(47-1800)$ & $312(70-330)$ & 0.89 \\
\hline $\begin{array}{l}\text { Serum ferritin at diagnosis } \mu \mathrm{gl}^{-1} \\
\text { (median and range) }\end{array}$ & $330(8-3599)$ & $320(8-3599)$ & $540(340-1432)$ & $330(300-660)$ & 0.09 \\
\hline Transfusion need at diagnosis (\%) & $61(69)$ & $52(67)$ & $5(100)$ & $4(80)$ & 0.12 \\
\hline Disease transformation $(n, \%)$ & $\begin{array}{r}\text { AML }(5,6) \\
\text { CMML }(1,1) \\
\text { RAEB-2 }(1,1)\end{array}$ & $\begin{array}{r}\text { AML }(5,6) \\
\text { CMML }(1,1) \\
\text { RAEB-2 }(1,1)\end{array}$ & 0 & 0 & 0.50 \\
\hline Number of deaths (\%) & $60(68)$ & $50(64)$ & $5(100)$ & $5(100)$ & 0.60 \\
\hline $\begin{array}{l}\text { Follow-up duration in months } \\
\text { (median and range) }\end{array}$ & $33(0-158)$ & $31(0-158)$ & $35(4-96)$ & $44.2(0.4-102)$ & 0.61 \\
\hline
\end{tabular}

Abbreviations: AML, acute myeloid leukemia; CMML, chronic myelomonocytic leukemia; F, female; JAK2, janus kinase 2; M, male; MCV, mean corpuscular volume; RAEB-2, refractory anemia with excess blasts-2.

${ }^{a} P$-values are for comparison of JAK2V617F-positive and -negative groups.

patients with leukemic transformation had received lenalidomide therapy and two had received erythropoiesis-stimulating agents therapy. The cause of death was documented in $29(48 \%)$ of the 60 patients who had died: 8 pneumonia, 7 cardiac failure, 4 cardiac arrest, 3 sepsis, 2 metastatic lung cancer and 1 each of metastatic breast cancer, myocardial infarction, stroke and lower gastrointestinal bleed. None of the deaths were directly related to iron overload.

\section{Survival, leukemic transformation rates and} prognostic variables

Median follow-up for the entire study population, including deceased patients, was 33 months (range, 0-158). During this period $60(68 \%)$ patients had died and 7 had documented disease transformation, that is, $5(5.7 \%) \mathrm{AML}$ and one case each of refractory anemia with excess blast- 1 and chronic myelomonocytic leukemia. The median time interval between diagnosis 
and leukemic transformation was 30 months (range, 8-39). None of the cases with disease transformation carried JAK2 or MPL mutations. Four of the five patients with leukemic transformation had additional cytogenetic abnormalities at the time of transformation, including del(7q). The number of patients with leukemic transformation was too small to allow formal analysis of leukemia-free survival.

Overall median survival was 66 months. Table 5 outlines the results of univariate and multivariable survival analyses regarding the impact of parameters at diagnosis on survival. Age $\geqslant 70$ years, red blood cell transfusion need and the presence of BM dysgranulopoiesis were identified as independent predictors of inferior survival. These three risk factors were then used to construct low- (no risk factors), intermediate- (presence of one

Table 3 Pathological and cytogenetic details of 80 patients with World Health Organization (WHO)-defined 'myelodysplastic syndrome with isolated $\operatorname{del}(5 q)^{\prime}$

\begin{tabular}{lc}
\hline Pathological and cytogenetic variable & Number ${ }^{\text {a }}$ \\
\hline Dyserythropoiesis (\%) & $28(35)$ \\
Dysgranulopoiesis (\%) & $16(20)$ \\
Dysmegakaryopoiesis (\%) & $69(86)$ \\
Dysplasia affecting a single cell line (\%) & $36(45)$ \\
Dysplasia affecting two cell lines (\%) & $27(34)$ \\
Dysplasia affecting all three cell lines (\%) & $6(7)$ \\
Abnormal metaphases with isolated del(5q) & $13(2-26)$ \\
(median, range) & \\
& \\
$5 q$ breakpoints (\%) & $2(2)$ \\
5q13q22 & $51(57)$ \\
$5 q 13 q 33$ & $3(3)$ \\
$5 q 15 q 31$ & $21(24)$ \\
5q15q33 & $4(5)$ \\
5q22q31 & $4(5)$ \\
$5 q 22 q 33$ & $3(3)$ \\
5q22q35 & \\
\hline aPlease note that bone marrow and peripheral smears were available \\
for pathological re-review in 80 patients; cytogenetic information was \\
available for re-review in 88 patients. Seven patients had no overt \\
evidence of myelodysplasia.
\end{tabular}

risk factor) and high (presence of two or three risk factors)-risk patient groups. The corresponding median survivals were 102, 52 and 27 months, respectively $(P=0.002$; Figure 1$)$. Of note, there was no significant association between survival and serum ferritin.

\section{Discussion}

The current study is unique in several aspects. First, it is one of few studies, possibly the only one, which strictly adhered to the 2008 WHO criteria in selecting study patients with 'MDS with isolated del $(5 q)^{\prime}$. This is not a trivial matter as patient selection methods greatly affect the interpretation of clinical outcome data and information on molecular phenotype. Second, this is

Table 5 Prognostic factors for survival in 88 patients with World Health Organization (WHO)-defined 'myelodysplastic syndrome with isolated del $(5 q)^{\prime}$

\begin{tabular}{lcc}
\hline Variable & $\begin{array}{c}\text { Univariate analysis } \\
\text { (P-value) }\end{array}$ & $\begin{array}{c}\text { Multivariable analysis } \\
\text { (P-value) }\end{array}$ \\
\hline Age & 0.004 & \\
Age $\geqslant 70$ years & 0.01 & 0.02 \\
Sex & 0.13 & \\
$\mathrm{Hb}$ & 0.58 & \\
$\mathrm{MCV}$ & 0.57 & \\
WBC & 0.86 & \\
$\mathrm{ALC}$ & 0.68 & \\
AMC & 0.97 & \\
Platelets & 0.2 & 0.01 \\
Serum ferritin & 0.21 & \\
Dyserythropoiesis & 0.76 & \\
Dysgranulopoiesis & 0.02 & 0.04 \\
Dysmegakaryopoiesis & 0.57 & \\
Transfusion need at & 0.04 & \\
presentation & &
\end{tabular}

Abbreviations: ALC, absolute lymphocyte count; AMC, absolute monocyte count; $\mathrm{Hb}$, hemoglobin; $\mathrm{MCV}$, mean corpuscular volume; WBC, white blood count.

Table 4 Clinical and pathological characteristics of patients with the World Health Organization (WHO)-defined 'myelodysplastic syndrome with isolated $\operatorname{del}(5 q)^{\prime}$ and associated $M P L$ mutations

\begin{tabular}{|c|c|c|c|}
\hline Variable & Patient 1 & Patient 2 & Patient 3 \\
\hline Age/sex & 76/F & 72/F & 75/F \\
\hline Molecular analysis & MPLW515L & $\begin{array}{l}\text { MPLW515L } \\
\text { JAK2V617F }\end{array}$ & MPLW515L \\
\hline Transfusion need at presentation & No & No & Yes \\
\hline Hemoglobin (g per $100 \mathrm{ml}$ ) & 11.0 & 11.3 & 7.6 \\
\hline MCV (fl) & 104 & 110 & 102 \\
\hline Leukocyte count $\left(\times 10^{9}\right.$ cells per $\left.I\right)$ & 5.5 & 3.2 & 3.8 \\
\hline Absolute lymphocyte count $\left(\times 10^{9}\right.$ cells per I) & 2.6 & 0.9 & 1.2 \\
\hline Absolute monocyte count $\left(\times 10^{9}\right.$ cells per I) & 0.6 & 0.8 & 1.0 \\
\hline Platelet count $\left(\times 10^{9}\right.$ cells per I) & 340 & 326 & 417 \\
\hline Serum ferritin $\left(\mu \mathrm{gl}^{-1}\right)$ & 340 & 230 & 3500 \\
\hline $5 q$ break point & $5 q 33$ & $5 q 33$ & $5 q 33$ \\
\hline BM dyserythropoiesis & Yes & No & No \\
\hline BM dysgranulopoiesis & Yes & No & No \\
\hline BM dysmegakaryopoiesis & No & Yes & Yes \\
\hline Disease transformation & No & No & No \\
\hline Survival (months) & 171 & 25 & 61 \\
\hline Status at last follow-up & Alive & Alive & Alive \\
\hline
\end{tabular}

Abbreviations: BM, bone marrow; JAK2, janus kinase 2; MCV, mean corpuscular volume; MPL, myeloproliferative leukemia virus oncogene (thrombopoietin receptor). 


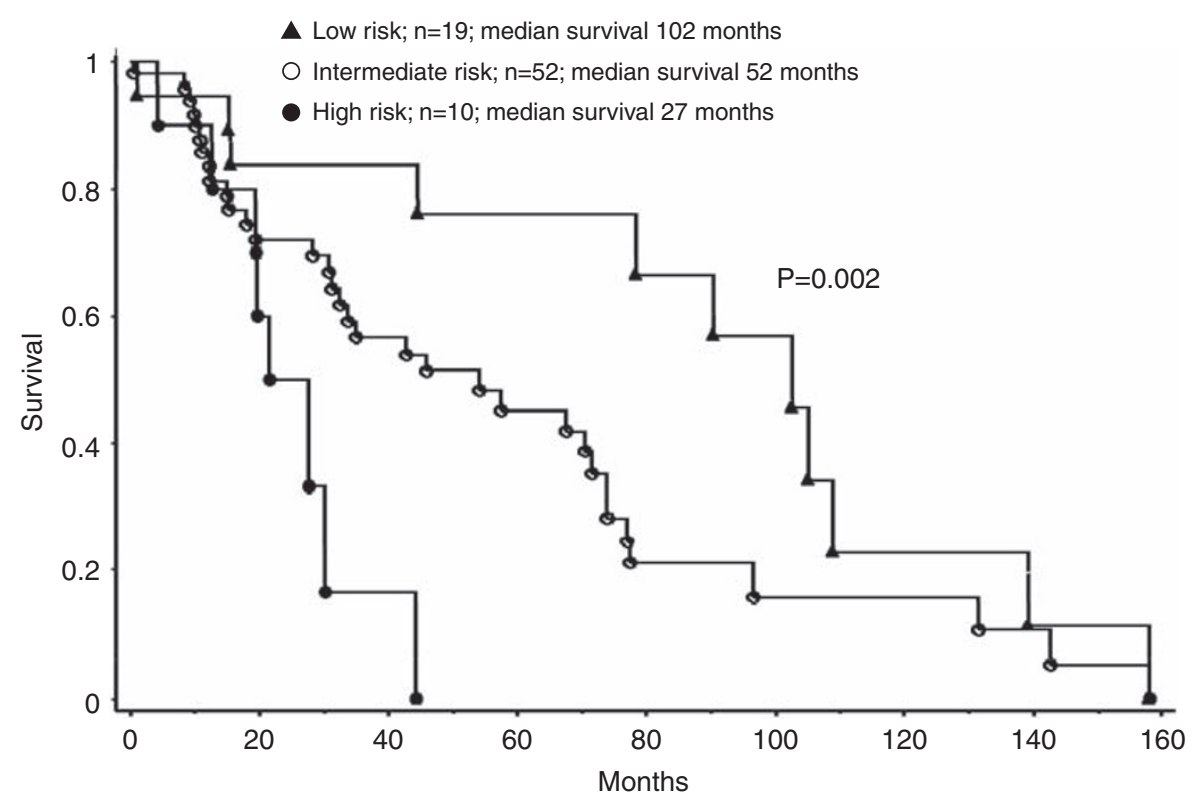

Figure 1 Kaplan-Meier survival curves for 88 patients with World Health Organization (WHO)-defined 'myelodysplastic syndrome with isolated del(5q)'. Overall median survival was 66 months. The figure shows median survival of patients belonging to three risk categories based on the presence of zero (low risk), one (intermediate risk) or $\geqslant 2$ (high risk) of the following risk factors: age $\geqslant 70$ years, transfusion need at presentation and the presence of bone marrow dysgranulopoiesis.

the largest single institutional study that has targeted a particular patient population and its clinical value is further enhanced by the availability of mature survival data. As a result, we were able to confirm the relatively indolent natural history of the disease and provide critical information regarding prognostic factors, the lack of survival impact from iron overload and baseline risk of leukemic transformation. The overall results of the current study are unlikely to have been affected by the $20 \%$ of the patients who received lenalidomide therapy, considering the fact that only $28 \%$ of them became transfusion-independent as a result.

In 1974, Van den Berghe et al. ${ }^{2}$ first described the ' $5 q-$ syndrome'. Subsequent studies have analyzed the clinical and pathological correlates for patients with MDS and del $(5 q)$, but often included patients with excess blasts or additional cytogenetic abnormalities. ${ }^{3-8}$ Most of these studies have reiterated the obvious fact that the presence of excess blasts or additional cytogenetic abnormalities adversely affects survival and increases the risk of leukemic transformation. ${ }^{3-8}$ What has been conspicuously missing is the information on long-term survival and prognostic factors limited to patients who fit strictly into WHO criteria for 'MDS with isolated $\operatorname{del}(5 q)$ '. The current study effectively addresses this particular issue by disclosing a more than 5-year overall median survival, which is even longer in patients below the age of 70 years and in the absence of both transfusion need at diagnosis and dysgranulopoiesis. We have previously reported on the adverse impact of dysgranulopoiesis on the survival of patients with MDS and isolated $\operatorname{del}(5 q){ }^{17}$ However, nearly half of the 68 patients in that particular study did not meet the WHO criteria for 'MDS with isolated del $(5 q)$ ', and the study population analyzed for survival included cases with refractory anemia with excess blast. ${ }^{17}$

The finding that dysgranulopoiesis has potential adverse prognostic impact in these patients is a unique observation that warrants further study and confirmation. As opposed to other prognostic variables, dysgranulopoiesis is a factor that can be easily determined at the time of diagnosis and needs to be evaluated as part of any 'MDS with isolated del $(5 q)^{\prime}$ BM evaluation. The presence of dysgranulopoiesis could also be a possible factor for the variably reported median survivals in previous studies of patients with del $(5 q)$. Regardless, it is currently not clear whether dysgranulopoiesis in 'MDS with isolated $\operatorname{del}(5 q)^{\prime}$ identifies patients with a different disease or represents an acquired histological marker of clonal evolution. ${ }^{17}$

JAK2V617F and MPLW515L are gain-of-function mutations that are characteristically associated with MPN and are only infrequently seen in patients with MDS. ${ }^{18,19}$ A previous multicenter study of 81 patients with MDS and isolated del(5q) identified six patients with $J A K 2 \mathrm{~V} 617 \mathrm{~F}$, all occurring in patients with less than 5\% BM blasts. The JAK2-mutated patients in this study displayed significantly higher leukocyte count and a trend toward a higher platelet count. However, it is not clear what constituted the comparison group, as the study population included 27 patients with excess blasts. ${ }^{20}$ In our study, there was no significant difference in blood counts or clinical outcome between patients with and without JAK2V617F. In MPN, the presence of $J A K 2 \mathrm{~V} 617 \mathrm{~F}$ has been associated with older age at diagnosis, higher hemoglobin level, leukocytosis and lower platelet count. ${ }^{21}$ Obviously, the small sample sizes in the del(5q)-related studies do not allow one to make statistically valid conclusions on the phenotypic effect of mutant JAK2 in that setting. Incidentally, a recent study looked into the clonal structure of JAK2V617F-mutated MDS with isolated del(5q) and showed that the $\operatorname{del}(5 \mathrm{q})$ and JAK2V617F clones were mutually exclusive. $^{22}$

The current study is the first to show the presence of MPL mutations in $\sim 4 \%$ of patients with 'MDS with isolated del $(5 q)^{\prime}$ and absence of the recently described $I D H 1$ and $I D H 2$ mutations. $^{23,24}$ None of the MPL-mutated patients displayed thrombocytosis. MPLW515L, MPLW515K and other exon 10 MPL mutations have been described in essential thrombocythemia and primary myelofibrosis with mutational frequencies that range from 3 to $15 \% .^{13,25-29}$ MPLW515L is the most frequent MPN-associated MPL mutation. In essential thrombocythemia, 
the presence of a MPL mutation has been associated with older age, lower hemoglobin level, higher platelet count, microvascular symptoms and a higher risk of post-diagnosis arterial thrombosis. ${ }^{29,30}$ In primary myelofibrosis, the associated factors were female sex, older age, lower hemoglobin level and a higher likelihood of becoming transfusion dependent. ${ }^{28}$ Again, the number of MPL-mutated patients in the current study was too small to allow making any comparative remarks.

With regard to $I D H$ mutations, they were first described in gliomas $^{31}$ and subsequently in AML. ${ }^{24,32,33}$ Mutational frequency of $I D H 1$ in primary $A M L$ is $\sim 9 \%$ and the mutations usually cluster with normal karyotype, although a possible association with trisomy 8 has been suggested. ${ }^{24}$ More recently, IDH2 mutations were also reported in primary AML. ${ }^{32,33} \mathrm{IDH}$ mutations have also been described in secondary AML, including patients with antecedent MPN or MDS. ${ }^{23,34,35}$ In the two most recent studies, IDH mutations were shown in more than $20 \%$ of patients with post-MPN AML, $\sim 4 \%$ of those with primary myelofibrosis and rarely in $\mathrm{PV}$ or essential thrombocythemia. ${ }^{23,35}$ To date, there is no information on the prevalence of $I D H$ mutations in MDS.

In December 2005, based on encouraging results seen with lenalidomide in the treatment of transfusion-dependent, lowerrisk patients with MDS and del(5q), the United States Food and Drug Administration (FDA) approved lenalidomide therapy for this indication. ${ }^{6,36}$ Patients who received lenalidomide achieved a red cell transfusion independence rate of $67 \%$ and a complete cytogenetic response rate of $45 \%{ }^{6}$ More recently, concerns have been voiced regarding drug-induced leukemogenicity, especially in those patients who do not respond to treatment. ${ }^{37}$ In one long-term study with a median follow-up of 40 months, $15(36 \%)$ of 42 lenalidomide-treated patients with low- or intermediate-risk MDS and $\operatorname{del}(5 q)$ had transformed to $\mathrm{AML}{ }^{37}$ This figure is much higher than expected in a patient population that fits the WHO criteria for 'MDS with isolated $\operatorname{del}(5 q)^{\prime}$. However, a substantial number of the lenalidomide-treated patients in the particular study displayed excess blasts at study entry and are therefore not comparable with the patient population in the current study. It is also interesting to surmise that the presence of dysgranulopoiesis could also be a contributing factor to disease progression in these lenalidomide-treated patients. A carefully designed randomized study is needed to resolve such issues. In the mean time, it would be interesting to query currently existing databases of lenalidomide-treated patients with MDS and estimate the risk of leukemic transformation in those patients who meet the WHO criteria for 'MDS with isolated del $(5 q)$ ', and compare them with the baseline risk established from the current study.

\section{Conflict of interest}

The authors declare no conflict of interest.

\section{References}

1 Tefferi A, Vardiman JW. Myelodysplastic syndromes. N Engl J Med 2009; 361: 1872-1885.

2 Van den Berghe H, Cassiman JJ, David G, Fryns JP, Michaux JL, Sokal G. Distinct haematological disorder with deletion of long arm of no. 5 chromosome. Nature 1974; 251: 437-438.

3 Holtan SG, Santana-Davila R, Dewald GW, Khetterling RP, Knudson RA, Hoyer JD et al. Myelodysplastic syndromes associated with interstitial deletion of chromosome 5q: clinicopathologic correlations and new insights from the pre-lenalidomide era. Am J Hematol 2008; 83: 708-713.
4 Giagounidis AA, Germing U, Haase S, Hildebrandt B, Schlegelberger B, Schoch $\mathrm{C}$ et al. Clinical, morphological, cytogenetic, and prognostic features of patients with myelodysplastic syndromes and del $(5 q)$ including band q31. Leukemia 2004; 18: 113-119.

5 Kantarjian H, O'Brien S, Ravandi F, Borthakur G, Faderl S, Bueso-Ramos $\mathrm{C}$ et al. The heterogeneous prognosis of patients with myelodysplastic syndrome and chromosome 5 abnormalities: how does it relate to the original lenalidomide experience in MDS? Cancer 2009; 115: 5202-5209.

6 List A, Dewald G, Bennett J, Giagounidis A, Raza A, Feldman E et al. Lenalidomide in the myelodysplastic syndrome with chromosome $5 q$ deletion. N Engl J Med 2006; 355: 1456-1465.

7 Mathew P, Tefferi A, Dewald GW, Goldberg SL, Su J, Hoagland $\mathrm{HC}$ et al. The 5q- syndrome: a single-institution study of 43 consecutive patients. Blood 1993; 81: 1040-1045.

8 Van den Berghe H. The 5q- syndrome. Scand J Haematol Suppl 1986; 45: 78-81.

9 Santana-Davila R, Holtan SG, Dewald GW, Ketterling RP, Knudson RA, Hanson CA et al. Chromosome $5 q$ deletion: specific diagnoses and cytogenetic details among 358 consecutive cases from a single institution. Leuk Res 2008; 32: 407-411.

10 Santana-Davila R, Tefferi A, Holtan SG, Ketterling RP, Dewald GW, Knudson RA et al. Primary myelofibrosis is the most frequent myeloproliferative neoplasm associated with del $(5 q)$ : clinicopathologic comparison of del(5q)-positive and -negative cases. Leuk Res 2008; 32: 1927-1930.

11 Dewald GW, Broderick DJ, Tom WW, Hagstrom JE, Pierre RV. The efficacy of direct, 24-hour culture, and mitotic synchronization methods for cytogenetic analysis of bone marrow in neoplastic hematologic disorders. Cancer Genet Cytogenet 1985; 18: $1-10$.

12 Shaffer LG, Tommerup N. An International System for Human Cytogenetic Nomenclature. S Karger: Basel, Switzerland, 2005.

13 Pardanani AD, Levine RL, Lasho T, Pikman Y, Mesa RA, Wadleigh M et al. MPL515 mutations in myeloproliferative and other myeloid disorders: a study of 1182 patients. Blood 2006; 108: 3472-3476.

14 Tefferi A, Lasho TL, Huang J, Finke C, Mesa RA, Li CY et al. Low JAK2V617F allele burden in primary myelofibrosis, compared to either a higher allele burden or unmutated status, is associated with inferior overall and leukemia-free survival. Leukemia 2008; 22: 756-761.

15 Watanabe T, Nobusawa S, Kleihues P, Ohgaki H. IDH1 mutations are early events in the development of astrocytomas and oligodendrogliomas. Am J Pathol 2009; 174: 1149-1153.

16 Nobusawa S, Watanabe T, Kleihues P, Ohgaki H. IDH1 mutations as molecular signature and predictive factor of secondary glioblastomas. Clin Cancer Res 2009; 15: 6002-6007.

17 Chen D, Hoyer JD, Ketterling RP, Tefferi A, Steensma DP, Holtan $\mathrm{SG}$ et al. Dysgranulopoiesis is an independent adverse prognostic factor in chronic myeloid disorders with an isolated interstitial deletion of chromosome 5q. Leukemia 2009; 23: 796-800.

18 Kilpivaara O, Levine RL. JAK2 and MPL mutations in myeloproliferative neoplasms: discovery and science. Leukemia 2008; 22: 1813-1817.

19 Tefferi A. JAK and MPL mutations in myeloid malignancies. Leuk Lymphoma 2008; 49: 388-397.

20 Ingram W, Lea NC, Cervera J, Germing U, Fenaux P, Cassinat B et al. The JAK2 V617F mutation identifies a subgroup of MDS patients with isolated deletion $5 q$ and a proliferative bone marrow. Leukemia 2006; 20: 1319-1321.

21 Vannucchi AM, Antonioli E, Guglielmelli P, Pardanani A, Tefferi A. Clinical correlates of JAK2V617F presence or allele burden in myeloproliferative neoplasms: a critical reappraisal. Leukemia 2008; 22: 1299-1307.

22 Sokol L, Caceres G, Rocha K, Stockero KJ, Dewald DW, List AF. JAK2(V617F) mutation in myelodysplastic syndrome (MDS) with del(5q) arises in genetically discordant clones. Leuk Res 2010; 34: 821-823.

23 Green A, Beer P. Somatic mutations of IDH1 and IDH2 in the leukemic transformation of myeloproliferative neoplasms. N Engl J Med 2010; 362: 369-370.

24 Mardis ER, Ding L, Dooling DJ, Larson DE, McLellan MD, Chen K et al. Recurring mutations found by sequencing an acute myeloid leukemia genome. N Engl J Med 2009; 361: 1058-1066. 
25 Pikman Y, Lee BH, Mercher T, McDowell E, Ebert BL, Gozo M et al. MPLW515L is a novel somatic activating mutation in myelofibrosis with myeloid metaplasia. PLoS Med 2006; 3: e270.

26 Schnittger S, Haferlach C, Beelen DW, Bojko P, Dengler R, Diestelrath A et al. Detection of three different MPLW515 mutations in $10.1 \%$ of all JAK2 V617 unmutated ET and $9.3 \%$ of all JAK2 V617F unmutated OMF: a study of 387 patients. ASH Annu Meet Abstr 2007; 110: 2546.

27 Vannucchi AM, Antonioli E, Pancrazzi A, Guglielmelli P, Di Lollo S Alterini $\mathrm{R}$ et al. The clinical phenotype of patients with essential thrombocythemia harboring MPL 515W $>\mathrm{L} / \mathrm{K}$ mutation. ASH Annu Meet Abstr 2007; 110: 678.

28 Guglielmelli P, Pancrazzi A, Bergamaschi G, Rosti V, Villani L, Antonioli $\mathrm{E}$ et al. Anaemia characterises patients with myelofibrosis harbouring Mpl mutation. Br J Haematol 2007; 137: 244-247.

29 Beer PA, Campbell PJ, Scott LM, Bench AJ, Erber WN, Bareford D et al. MPL mutations in myeloproliferative disorders: analysis of the PT-1 cohort. Blood 2008; 112: 141-149.

30 Vannucchi AM, Antonioli E, Guglielmelli P, Pancrazzi A, Guerini $\mathrm{V}$, Barosi $\mathrm{G}$ et al. Characteristics and clinical correlates of MPL $515 \mathrm{~W}>\mathrm{L} / \mathrm{K}$ mutation in essential thrombocythemia. Blood 2008; 112: $844-847$.

31 Parsons DW, Jones S, Zhang X, Lin JC, Leary RJ, Angenendt P et al. An integrated genomic analysis of human glioblastoma multiforme. Science 2008; 321: 1807-1812.

32 Ward PS, Patel J, Wise DR, Abdel-Wahab O, Bennett BD Coller $\mathrm{HA}$ et al. The common feature of leukemia-associated $\mathrm{IDH} 1$ and $\mathrm{IDH} 2$ mutations is a neomorphic enzyme activity converting alpha-ketoglutarate to 2-hydroxyglutarate. Cancer Cell 2010; 17: 225-234.

33 Gross S, Cairns RA, Minden MD, Driggers EM, Bittinger MA, Jang HG et al. Cancer-associated metabolite 2-hydroxyglutarate accumulates in acute myelogenous leukemia with isocitrate dehydrogenase 1 and 2 mutations. J Exp Med 2010; 207: 339-344.

34 Abdel-Wahab O, Manshouri T, Patel J, Harris K, Yao J, Hedvat C et al. Genetic analysis of transforming events that convert chronic myeloproliferative neoplasms to leukemias. Cancer Res 2010; 70: 447-452.

35 Pardanani A, Lasho T, Finke C, Mai M, McClure R, Tefferi A. IDH1 and IDH2 mutation analysis in chronic and blast phase myeloproliferative neoplasms. Leukemia 2010 (in press).

36 List A, Kurtin S, Roe DJ, Buresh A, Mahadevan D, Fuchs D et al. Efficacy of lenalidomide in myelodysplastic syndromes. N Engl J Med 2005; 352: 549-557.

37 Gohring G, Giagounidis A, Busche G, Kreipe HH, Zimmermann M, Hellstrom-Lindberg E et al. Patients with del(5q) MDS who fail to achieve sustained erythroid or cytogenetic remission after treatment with lenalidomide have an increased risk for clonal evolution and AML progression. Ann Hematol 2010; 89: 365-374.

(c) This work is licensed under the Creative Commons Attribution-NonCommercial-No Derivative Works 3.0 Unported License. To view a copy of this license, visit http:// creativecommons.org/licenses/by-nc-nd/3.0/ 